\title{
Online biofilm monitoring
}

\author{
Peter Janknecht \& Luis F. Melo* \\ LEPAE or Department of Chemical Engineering, Faculty of Engineering, University of Porto, P-4200465 \\ Porto, Portugal (*author for correspondence: e-mail: Imelo@fe.up.pt)
}

Key words: biofilm monitoring, online monitoring

\begin{abstract}
A review of online biofilm monitoring techniques is presented focusing on methods based on differential turbidimetry, light scattering, heat transfer, pressure drop, real-time measurement of metabolic products, image analysis, radiation signals (spectroscopy, fluorometry, photoacoustic spectroscopy, etc.), electric and mechanical (vibration) signals. The different methods are compared in terms of their applicability to practical situations and the know detection limits are reported.
\end{abstract}

\section{Introduction}

One of the most remarkable and best documented features of biofilms is the fact that they can form under extremely diverse conditions, which becomes a dominating consideration once we attempt to monitor biofilms.

On the one hand, the environments in which biofilms form can differ in each and every chemical and physical parameter. Monitoring methods that work perfectly in one application therefore can be completely useless in another.

On the other hand, biofilms can be desirable, unwelcome or even harmful with corresponding consequences for the detection scale: the detection of individual microorganisms in clean environment at bench scale presents different challenges than monitoring the growth of a voluminous bacterial lawn with a complex biocenosis in natural systems or technical applications.

As a result we are confronted with the curious fact that a confusingly high number of analytical methods are applied to biofilm monitoring in general, while the choice of feasible methods in a given application can be extremely limited.

This chapter is intended to give an overview of both traditional and recent online methods in the field and how they work. By doing so, however, it may serve the purpose of giving ideas for applications that might demand completely new approaches. After all, there are as many methods of biofilm monitoring as there are biofilms.

\subsection{Further reading}

The online biofilm monitoring methods presented here may be applied in the laboratory as well as in industry or field research. Some methods, however, require equipment or operating conditions that can limit their practical applicability to the laboratory. These will receive less attention here, since they are discussed extensively in the recent literature. Readers who are interested in them are therefore referred to the following two review papers:

In 1995 Nivens et al. presented a comprehensive selection of advanced online biofilm monitoring methods available at that time, titled "Continuous non-destructive monitoring of microbial biofilms: a review of analytical techniques". The methods presented in that paper are still state of the art and will also be depicted in this chapter, supplemented by more recent relevant literature.

Wolf et al. (2002) published a review on "Optical and spectroscopic methods for biofilm examination and monitoring". The spectroscopic part of this work includes several on-line methods and their detailed descriptions.

One major literature source for this chapter was the International Specialized Conference on 
Biofilm Monitoring, held in March 2002 in Porto, Portugal. Information presented in this event included technologies that only evolved in the last years, as well as some less sophisticated and more traditional methods, which are still in use and therefore are also mentioned here (cf. also Water Science and Technology, vol. 47, no. 5, 2003).

\subsection{Basic considerations}

First of all, it might clarify some facts to quote a conclusion by Lewandowski (2003): "Monitoring biofilms can provide insight into fundamental biofilm processes. However, one needs to be fully aware of what is actually measured, and how the measured quantities correlate with fundamental features describing biofilm systems, like rates of biofilm accumulation and microbial activity. It remains unclear which parameters of biofilm process should be monitored to gain such knowledge".

From an abstract point of view, all online biofilm monitoring techniques are based on some kind of signal obtained from the biofilm under investigation. Usually these signals constitute some kind of energy transfer, such as radiation (including light), acoustic waves, electrical fields, electric current, or heat transfer. A few of these signals are actively emitted by the biofilm, such as bioluminescence, but the vast majority is a passive response to input signals that were generated by the monitoring equipment. In the actual process of biofilm monitoring these input signals are transmitted to the investigated surface, modified by the biofilm (if present) and its environment, detected by specific sensors and usually processed in more or less sophisticated ways. It is in the modification of the signal, that the biofilm leaves its characteristic footprint. Signal features that may be modified include:

- intensity of light (Differential Turbidity)

- intensity of sound (Ultrasonic FrequencyDomain Reflectometry)

- color/wavelengths (Bioluminescence, Fluorometry, Spectroscopy)

- mechanical resonance frequencies (Quartz Crystal Microbalance)

- electrical capacitance (Dielectric Sensor)

- electrical conductivity (Electrochemical Electrodes)

- light refraction indices (Surface Plasmon Resonance)
- friction (Pressure Drop)

- thermal resistance (Heat Transfer Coefficient)

- optical input signals that are being modified into acoustic output signals (Photoacoustic Spectroscopy).

\section{Deposit measurements}

Under certain conditions, one can safely assume that any deposit occurring on a solid surface must be a biofilm of more or less known composition, so that it suffices to detect the presence and thickness of the deposits without determining other characteristics. This is often the case, for example, in industrial processes where biodegradable matter of known composition is suspended or dissolved in water.

Several features that biofilms share with any other deposits can be utilized for such measurements, such as light absorption, thermal insulation, or increased friction to moving fluids. Methods like these lend themselves especially to applications, where the main interest in biofilms is actually related to their ability to obstruct heat transfer or cause excessive pressure drops.

\subsection{Differential turbidity (DTM)}

A very simple yet efficient method using the absorption and scattering of light by a biofilm has been described by Klahre \& Flemming (2000). The setup essentially consisted of two turbidity meters monitoring the turbidity in a whitewater stream of a paper mill process. While the glass windows of one turbidity meter were regularly cleaned by a water jet, the other one accumulated deposits which increased the measured turbidity value. The difference of the two instruments' readings thus corresponded to the accumulation of deposits between them. The method was not able to detect biofilm thickness of less than $0.1 \mathrm{~mm}$, which might be limiting in some applications. Practical problems concerning the water jet cleaning process can however occur.

A very similar technique has been presented by Wetegrove \& Banks (2002), using mechanical wipers instead of water jets for cleaning the reference detector. The same author also presents a similar method, in which light is conducted 


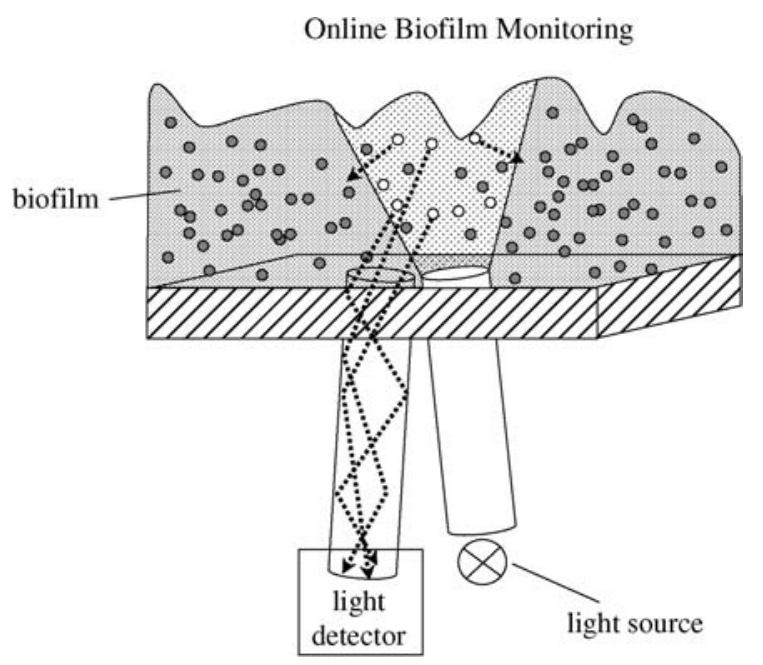

Figure 1. Scheme of FOS.

through fiber optics and divided into two separate beams. The first beam passes through a liquid streaming in a transparent tube, while the second one only passes through the free flowing system. Since the biofilm can only form inside the tube, the difference in light reduction between the two beams is considered to correspond to its growth.

\subsection{Fiber optical device (FOS)}

A similar technique is based on a fiber optical probe, which applies light from below to the surface of its head and detects how much is scattered back from any deposits. It has been successfully applied in a brewery water pipeline system and can detect bacterial colonization above $10^{5}$ cells cm $\mathrm{cm}^{-2}$ (Tamachkiarow \& Flemming 2003), see Figure 1. This technique is not appropriate for thick biofilms.

\subsection{Heat Transfer}

The build up of a biofilm on a surface introduces an additional resistance to heat transfer through the system that can be assessed if the heat flux and the wall and fluid temperatures are known. This is the idea behind the heat transfer monitor in Figure 2, which is composed of two flow channels: one for the test fluid (that is, the one causing biofilm formation) and the other for the auxiliary fluid (the heat source). A flat wall (metal or other material) separates the two flow channels. One thermocouple $\left(T_{1}\right)$ is immersed in the test liquid and two are imbedded in the wall $\left(T_{2}, T_{3}\right)$ in the

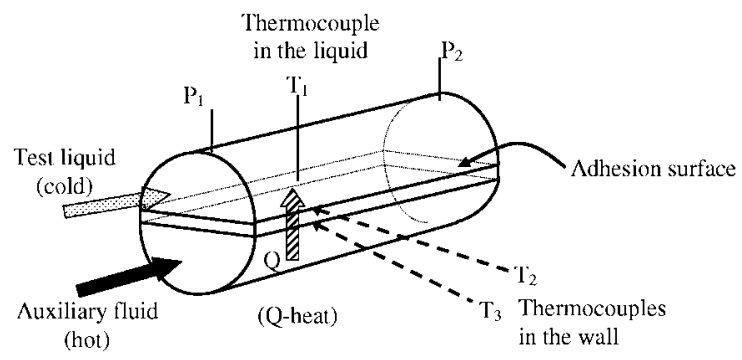

Figure 2. Heat transfer monitor.

same radial position. Thus, local heat transfer coefficients can be obtained along the surface. Pressure drop $\left(\Delta P=P_{1}-P_{2}\right)$ is also measured in order to take into account the effect of biofilm roughness on the convective heat transfer coefficient of the test liquid.

Some monitors use an electrical resistance as a heat source instead of the hot fluid: if the heat flux provided by the electrical resistance is known, only one thermocouple is needed in the wall.

Many other small heat transfer monitors have been reported in the literature on fouling/biofouling of heat exchangers (Knudsen 1981; Chenoweth 1988) which are usually connected to a side-stream in an industrial plant. One of the most frequently used monitors is the annular test section, composed of two concentric tubes, the outer one being made of transparent plastic to allow visual observation. The fluid that causes biofouling circulates in the annular core, while the heat source (an electrical resistance heater, or a hot fluid) is placed in the inner cylinder. Thermocouples are located in the wall of the inner tube and in the test fluid.

Heat transfer techniques are obviously well fitted for monitoring biofouling in heat exchangers, such as in industrial cooling water systems and in power plant condensers. The monitors can also be used to follow biofilm build up in other water flow systems, since there is, in general, no need for high heat fluxes to measure the thermal resistances (temperature differences between the water and the wall can be small), and therefore biofilms will not be affected by temperature.

The main limitations of the heat transfer monitoring techniques include: firstly, the hydrodynamic pattern in these monitors may not always represent the actual situation occurring in the heat exchanger tubes or channels, secondly, the measurement of wall temperatures is generally subject 
to experimental uncertainties that are difficult to avoid; thirdly, the technique does not have enough sensitivity to detect the initial attachment of the microorganisms. It is not probable that it can measure the formation of the first biofilm monolayer, because the additional thermal resistance is still very low at that stage (a biofilm of $10 \mu \mathrm{m}$ thickness will increase the overall thermal resistance by $1-1.5 \%$, which is not clearly detected using heat transfer measurements). Also, the accuracy of the technique is limited by the fact that the areas of the clean surface and of the biofilm surface are not equal, on account of the roughness and heterogeneity of the microbial layer.

\subsection{Pressure drop}

When a biofilm layer attaches to the wall of a smooth pipe, it causes two direct effects on the liquid flow:

(i) it increases the roughness of the surface in contact with the liquid;

(ii) it reduces the cross-sectional area of the flow passage.

These effects can be detected by measuring the pressure drop of the liquid through the pipe. The first colonies of microorganisms that adhere to the surface cause an increase in the friction factor, because the surface becomes "rougher" (meaning that the thickness of the viscous sublayer is less than the height of the roughness peaks). Increased pressure drop can also be due to reduced channel crosssection, to biofilm oscillations (related to the viscoelastic nature of the microbial layer, that absorbs energy from the fluid) and to the presence of filaments (streamers) on the biofilm-liquid interface.

Pressure drop measurements are frequently used in industry to monitor fouling of heat exchangers, on account of the simplicity and low cost of the method. They can also be applied in tubes other than heat exchangers.

Attachment of microbial colonies of $10 \mu \mathrm{m}$ on the surface would tend to increase the friction factor and, therefore, the pressure drop by $10-15 \%$. However, since the thickness of the viscous sublayer in tubes is often higher than $10 \mu \mathrm{m}$, the microbial aggregates will not protude out of the sublayer and the value of the friction factor will not be affected by the presence of the colonies. Characklis et al. (1990) reported experi- mental results that indicate significant changes in the friction factor due to biofilms only after a "critical thickness" of around $30-35 \mu \mathrm{m}$ is attained.

\subsection{Thickness}

Some authors (Pinheiro et al. 1988) used a needle connected to the tip of a micrometer to measure the thickness of biofilms. The thickness is given by the difference between the micrometer readings when the needle touches the liquid-biofilm interface and when it touches the substratum (the base surface). More recently, Freitas dos Santos \& Livingston (1995), proposed a new technique using a light source which illuminates the biofilm and projects its amplified cross-section image on a screen. The estimated error is $\pm 10 \mu \mathrm{m}$.

\section{Metabolic products}

All living organisms leave tracks in their environment in the shape of substances produced by their metabolism. In cases where these substances are characteristic and detectable, they may be utilized to monitor the occurrence of the corresponding organisms. Although this procedure does not inherently discriminate between biofilms and suspended biomass, the presence of the latter may be excluded or neglected under certain conditions and the results solely referred to biofilm activity.

The metabolic products in question may be liquid or gaseous. Bastos et al. (2002), for example, reported a work in which aqueous chloride concentration was continuously measured by sequential injection analysis (SIA) to monitor the performance of a trickling reactor for monochlorobenzene-removal from air. Vanhooren et al. (2002) treated synthetic wastewater with the same kind of reactor and assessed its operation by paramagnetic online analysis of $\mathrm{O}_{2}$ and $\mathrm{CO}_{2}$ in the off-gas. Pereira et al. (2003) monitored the performance of anaerobic granular sludge by its methane yield, while Kappelhof et al. (2002) presented the specific oxygen consumption rate (SOCR) for the monitoring of membrane-biofouling in tap water which is similar to the approach by Carrión et al. (2003) who measured the overall biofilm respiration rate in a nitrifying fixed bed reactor. No explicit detection limit is named in 
the publication, but the correlation of SOCR and ATP-measurements in this work might indicate that it is in the range of one nanogram of ATP per $\mathrm{cm}^{2}$ of surface.

\section{Computerized image analysis}

In theory there are many ways of using computerized image analysis in the field of biofilm monitoring. Determining the portion of white and black pixels on a picture taken from a water container's illuminated window (Le Bras et al. 1998) is probably the simplest one. In more complex methods, especially designed and programmed software can actually recognize individual cells and biofilm structures. Nivens et al. already pointed out the potential of this method for industrial application in 1995, and work on this field continues (Heydorn et al. 2000; Xavier et al. 2000, 2003). But even though the advances in data processing equipment must be expected to strongly favor this technology, there is no indication of its use in online biofilm monitoring, except for laboratory application.

The obvious drawbacks of microscopic methods include the necessity to have sensitive (and expensive) microscopy and image acquisition equipment present on site, with image analysis hardware and software also either present or connected online. To further complicate the matter, the appropriate application of dyes, which forms an essential part of most microscopic methods, is difficult to integrate in an automated setup. Furthermore, the dye application often affects the investigated biological samples, thus rendering impossible any continuous monitoring of fixed samples like biofilms. Nevertheless, the use of microscopic methods in industrial or field-research online biofilm monitoring should not be generally excluded at the outset. For the backgrounds of these methods the reader is referred to the review by Wolf et al. (2002).

\section{Detection of radiation signals}

A large number of online biofilm monitoring methods is based on the detection of visible, infrared, and ultraviolet light as well as radio frequencies, all of which may be physically summarized as electromagnetic radiation signals (Figure 3).

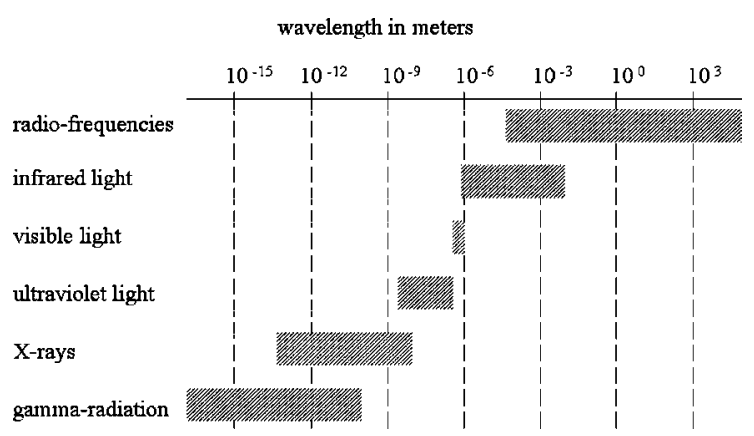

Figure 3. Electromagnetic radiation wavelengths.

In order to understand the following monitoring methods it might be useful to recall some basic principles of the possible interactions between electromagnetic radiation and atoms: Every atom consists of an inner core or nucleus and an outer electron-shell, in which exist discrete and characteristic energy levels for the individual electrons. When atoms combine to molecules, some of these energy levels change and can become a unique characteristic for this special molecule or chemical group within a molecule.

The electrons of the electron-shell have the ability to jump between these levels, but only when they receive or give away a portion of energy which precisely corresponds to the difference of the respective energy levels (minus a certain friction loss, as even electrons get nothing for free).

One way in which this energy can be transported to or from the electron is a radiation impulse or photon. The shorter the wavelength of the radiation, the higher is the energy of its photons. Therefore, since certain energy portions are characteristic for certain molecules and wavelength corresponds to energy portion, the wavelengths of radiation emitted or absorbed by a molecule are also characteristic for it.

\subsection{Bioluminescence}

Among the radiation signals utilized for biofilm detection, bioluminescence signals are the only ones that are spontaneously generated by the biomass without requiring any input of external energy. These signals consist of light which certain organisms are capable of actively emitting, an example being the notorious sea-glowing caused by planktonic microorganisms. In these cases the 
energy that excites the electrons is provided by an enzymatic reaction incorporated in the metabolisms. It is possible to utilize these usually weak light signals to detect the presence of such organisms.

This active light emission, however, is a function, among others, of the respective organisms' metabolic activity, which in turn depends on numerous environmental factors. Therefore bioluminescence sometimes enables the direct monitoring of such factors. On the other hand, however, quantitative determination of biomass by bioluminescence, as reported by Angell et al. (1993), is only possible under favorable conditions.

Bioluminescence is limited to relatively few organisms that naturally emit light or to such that have been genetically manipulated to do so. In most industrial or field research applications, however, the presence of non-light-emitting microorganisms must be anticipated. The validity of bioluminescence detection for bulk biofilm monitoring under non-laboratory conditions therefore is limited.

\subsection{Fluorometry}

Another way for electrons to emit radiation is after absorbing a photon from some incoming radiation, a process which is referred to as (auto-) fluorescence. Due to the discrete energy levels, however, only incoming radiation of the right wavelength (i.e. photons with the right energy portion) can accomplish this step. Furthermore, since some of the energy is lost between the transitions, the emitted photon always has a longer wavelength than the absorbed, thus the wavelengths that a given molecule absorbs are not the same as those it emits. Nevertheless, both the absorbed and the emitted wavelengths are equally characteristic for a given molecule and can be utilized for its detection. Simply speaking, the analysis of light emitted by a substance is called fluorometry, while that of absorbed radiation is called spectroscopy. Both can be and have been applied to biofilm monitoring.

Care must be taken however, with the radiation to be absorbed: high-energy radiation such as ultraviolet and X-ray can seriously damage the biofilm by photodecomposition of molecules, thus rendering continuous monitoring on one sample impossible. Low-energy radiation, however, such as visible light, the lower infrared spectra, and radio frequencies can often be utilized continuously as long as their intensity does not create a local temperature increase in the sample that affects the parameters under investigation.

Fluorometry, the detection of light emitted by molecules, can target different biomolecules. Some of those allow for quantitative detection of biomass (e.g. tryptophan, Saxena et al. 2002a, b) or the presence of biofilm (exopolysaccharides or EPS, Saxena et al. 2002a, b), whereas others can yield information about the current activity of the biomass (e.g. NAD(P)H) and cell growth (e.g. pyridoxine, Wolf et al. 2002). When using fluorometry as a biofilm monitoring method it is important to keep in mind several factors that are capable of falsifying the results, such as the possibility of the incoming radiation actually damaging the light emitting molecules (photodegradation), the excited electron state being quenched by other processes without emitting the corresponding light (quenching), absorption of certain emitted wavelengths by other molecules (cascade effect), or their scattering by particles within or around the biofilm. Furthermore, environmental factors like mixing fluid, oxygen concentration, temperature and $\mathrm{pH}$ have been reported to influence fluorometry readings (Nivens et al. 1995; Wolf et al. 2002).

Conventional fluorometry is utilized by applying a band of radiation wavelengths to a sample and detecting the response at a given wavelength. For radiation in the optical range, a flexible optical fiber probe can be used to transmit both exciting and emitted signals, making for simplified application (Saxena et al. 2002a).

Due to the above mentioned restrictions this one-dimensional approach is often limited to laboratory applications with well defined environmental conditions, rather than natural or industrial systems.

One possible improvement, which might help overcome some of these problems, evolved in the last years and consists in the use of so called twodimensional wavelength scans. Like in conventional fluorometry a band of wavelengths is used to excite the sample's molecules; the detection of responses, however, is extended from just one to a complete range of wavelengths. Much more complex patterns of input-response relationships can be detected this way, which can be used to com- 
pensate some of the side-effects mentioned earlier. The amount of data involved in these twodimensional scans, however, requires considerable computing for the necessary pattern recognition (Wolf et al. 2003). The review by Wolf et al. (2002) contains an overview of such approaches, some of which might have the potential to expand the applicability of fluorometric methods considerably.

\subsection{Spectroscopy}

While fluorometry detects the response of molecules excited with external radiation, spectroscopy methods ignore possible responses and rather measure how much of the exciting radiation is absorbed at which wavelength. As described earlier, both the wavelengths of absorbed and emitted radiation are characteristic for a certain molecule.

With respect to what was stated about twodimensional fluorometry, spectroscopic methods are inherently one-dimensional, since for each applied radiation wavelength there can only be one value of absorption. On the other hand spectroscopy does not rely on the actual emission of new photons from the investigated molecules with all the possibly interfering processes described above, but rather concentrates on the detection of the very signal that is emitted by the monitoring equipment itself.

Scattering of radiation, which can have a very disturbing effect on the response radiation in fluorometry, can be compensated in spectroscopy by scanning the absorption spectra of different wavelengths and determining the wavelengthindependent loss in light intensity. In fluorometry this method of compensation is not applicable, because the emitted signals' intensities are unknown and only cover individual wavelengths. The same, of course, applies to spectroscopic methods that are limited to one wavelength, so that discrimination between weak response and scattering is impossible.

The wavelength range commonly used for biofilm monitoring is the infrared (IR). One feature of infrared light, however, is that water molecules, which must be accounted for in aqueous biofilm monitoring, also absorb it.

Scanning of the IR absorption in a sample at different wavelengths yields a characteristic "fin- gerprint" of certain wavelengths in which the radiation has been absorbed stronger than in other ones (Wolf et al. 2002). In a complex system such as organic cells, the bulk absorption spectra is made up by superimposing the individual absorption spectra of all the different molecules and functional groups present in the sample.

The measuring of absorption spectra can be done either by scanning different wavelengths consecutively (as done by so called "dispersive" instruments), or by applying and monitoring a whole wavelength-range of radiation simultaneously. For that aim the input-radiation is modulated, so that from the known modulation and the detected radiation output the actual absorption at the individual wavelengths can be computed mathematically using Fourier-transformations of the signal. Therefore this method is named "FT-IR" (Nivens et al. 1995)

Nevertheless, simple IR-absorption measurements with only one wavelength can also be usefiul. Tinham \& Bott (2003) reported the use of a simple IR-light source and detector on opposite sides of a transparent tube (a setup similar to the Differential-Turbidity method described above). This method was sufficient to monitor the formation of biofilms with thicknesses in the range above $0.05 \mathrm{~mm}$. The reported work was limited to pure cultures of Pseudomonas fluorescens, but the authors consider industrial applications possible e.g. for biofilm control.

\subsection{Attenuated total internal reflection (ATR)}

Attenuated Total Internal Reflection Spectroscopy with Evanescent Waves (ATR-EW) in biofilm monitoring has been described by Nivens et al. (1995), as a sampling technique used in IR-spectroscopy. The core of the ATR-equipment is a transparent solid body called internal reflection

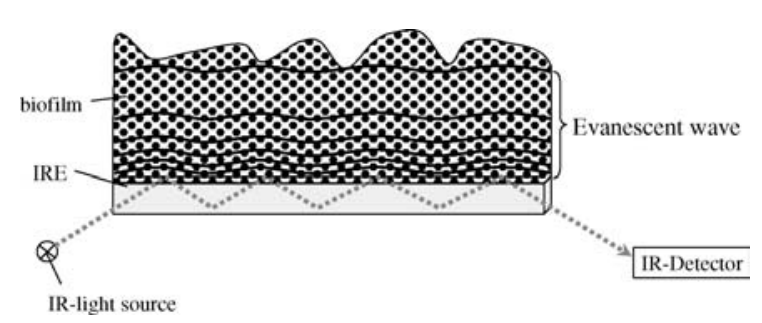

Figure 4. Scheme of ATR with Evanescent Waves (ATR-EW). 
element (IRE). A beam of infrared-radiation can travel through this element by being reflected on the inside of the solid material's surface (Figure 4). On each reflection, however, a part of the radiation "leaks out" of the element into the surrounding media. This radiation forms a standing or so-called evanescent wave, the intensity of which decreases exponentially with the distance from the IRE surface. Within the reach of this wave its radiation can be absorbed, which in turn is detectable as a decrease in the radiation intensity within the internal reflection element (Nivens et al. 1995).

The scanning of different radiation wavelengths in the IRE yields similar characteristic absorption patterns, just like the spectroscopic methods described above. Thus it is possible to detect the presence of different substances within the range of the evanescent wave, including those related to biofilms, from its substrate side.

One feature of ATR-Spectroscopy is that the geometrical range of detection is limited to a thin layer next to the IRE's surface (approximately $1 \mu \mathrm{m}$ ), thus efficiently eliminating interference from the bulk liquid, but also excluding the monitoring of thicker biofilms. The biofilm detection limit of this method was estimated to be $5 \times 10^{5}$ cells cm $\mathrm{cm}^{-2}$. Apparently it was only used in lab-scale so far.

An alternative application of ATR in biofilm monitoring uses surface plasmon resonance (ATRSPR), which is a phenomenon occurring when light is reflected by thin metal films. A fraction of the light can interact with the delocalized electrons in the metal film (plasmon) thus reducing the intensity of the reflected light. The precise angle of incidence at which this occurs is determined by several factors, including the refractive index of the matter next to the metal film's backside. Since the presence of exopolysaccharides and microorganisms in water can increase its refractive index, a loss in intensity of the reflected light can be correlated to the presence of a biofilm.

Like in ATR-spectroscopy the detection range of ATR-SPR is very short, thus focusing this method on the monitoring of very thin biofilms. Unlike in ATR-spectroscopy, however, only the presence of biofilms is detected without distinguishing between their individual components.

Leitz et al. (2002) used a high-index glass prism with a thin aluminum coating as IRE. Only one wavelength of light was utilized $(\Delta=632.8 \mathrm{~nm})$ and the SPR-patterns for different angles were determined. The growth of a pure Cytophagabiofilm caused these SPR-patterns to shift to higher angles. Philip-Chandy et al. (2000) used a plastic optical fiber of $1 \mathrm{~mm}$ diameter as IRE and applied light from a laser diode $(\Delta=660 \mathrm{~nm})$. This device was tested in a pilot plant simulating a cooling tower in parallel with a thermal flux sensor (see Section 2.3). While the detection limit of the latter was only reached after 14 days, the plastic optical fiber sensor displayed the first change in signal as early as 4 days, which seems to indicate its feasibility as an early warning sensor. Some correlation between biofilm buildup and the sensor signal was found, but a quantification of the detected biofilms by other methods was not reported.

A third method belonging to this group of techniques is the Total Internal Reflection Microscopy (Göransson \& Trägårdh 2000), in which the evanescent wave merely serves the purpose to illuminate the biofilm for microscopic observation. Thus the limitations mentioned in the section on microscopic methods also apply to this one.

\subsection{Nuclear magnetic resonance (NMR) spectroscopy}

A number of atoms such as ${ }^{1} \mathrm{H},{ }^{19} \mathrm{~F},{ }^{23} \mathrm{Na}$ and ${ }^{31} \mathrm{P}$, as well as some rare isotopes like ${ }^{13} \mathrm{C}$ and ${ }^{15} \mathrm{~N}$ possess the property of magnetic spin. This means that the nuclei of these atoms can be oriented in a magnetic field, and excited by an impulse of radiofrequency (rf) radiation. After excitation they return to their equilibrium state, in turn emitting rf-signals, a process somewhat similar to the absorption and emission of radiation by electrons as described in the fluorometry section. The emitted rf-pulses can be detected by an NMR probe and yield certain information about the respective sample, such as the concentration and even movements (flow, dispersion and diffusion) of the emitting nuclei. From these parameters further information can be deduced such as the porosity of a sample. The combination of results obtained from different spin nuclei (e.g. ${ }^{1} \mathrm{H},{ }^{23} \mathrm{Na}$ and ${ }^{31} \mathrm{P}$ ) can yield information about metabolic conditions of a bio-system (As \& Lens 2001; Wolf et al. 2002).

The use of ${ }^{13} \mathrm{C}$ and ${ }^{15} \mathrm{~N}$ for the same purpose, though theoretically possible, is practically ob- 
structed by the fact that only an infinitesimal fraction of naturally occurring carbon and nitrogen is made up of these isotopes, whereas ${ }^{1} \mathrm{H},{ }^{23} \mathrm{Na}$ and ${ }^{31} \mathrm{P}$ each constitute the vast majority of the existing atoms of the respective species.

It is possible to carry out sequences of NMRmeasurements in the presence of magnetic fields with linear gradients and derive a certain space resolution by Fourier transformation from these results. This process, called Magnetic Resonance Imaging (MRI), enables the generation of twodimensional images of a sample (As \& Lens 2001).

Though the potential of NMR-application in field studies has been recognized, the use of NMR outside laboratories is not common. The reasons for that might have to do with the unhandy and expensive equipment, similar as was discussed for microscopic techniques.

\subsection{Photoacoustic spectroscopy (PAS)}

Instead of measuring how much radiation is absorbed by a given sample, as is done in light spectroscopy, photoacoustic spectroscopy measures one effect of this absorption. A short radiation impulse, usually from a laser, is absorbed by a suitable substance inside the sample, generating heat, which in turn causes a physical expansion and a subsequent pressure or acoustic wave that can be detected e.g. by a piezoelectric film acting as a microphone (Figure 5). The sensor utilized for PAS-biofilm monitoring consists of a prism which simultaneously serves as a surface for biofilm growth and a transmitter, both for the radiation impulse to the biofilm and the acoustic wave traveling back to the piezoelectric film attached at the back of the prism.

One interesting feature of this method is that the relatively slow speed of the acoustic waves enables a certain depth-resolution (about $10 \mu \mathrm{m}$ ) of the measurements, based on the time delay be-

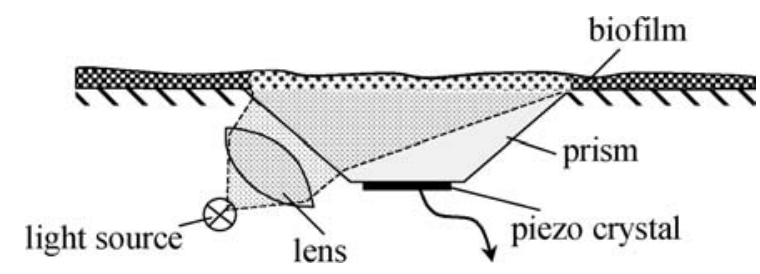

Figure 5. Scheme of a PAS-sensor. tween laser pulse and acoustic wave. Though it might be questionable whether the speed of sound is identical in bulk liquid and biofilm, this method facilitates assessment of thickness and homogeneity of a biofilm, and detect growth or detachment over time (Schmidt et al. 2001, 2002).

Furthermore, by comparing the responses to pulses of different radiation wavelengths one can distinguish between different biofilm components such as water, pigments, and carbohydrates and further detect their relative distribution within the biofilm (Schmid et al. 2003).

\section{Electric signals}

\subsection{Electrochemical techniques}

There are several ways to obtain an electric signal from micro-organisms indicating their presence. Most of these methods consist in bringing two or more electrodes in contact with the aqueous phase and detecting changes in their behavior such as their actively built up electrical potential or their passive response to the application of certain voltage or current signals.

The use of electrodes is common in monitoring so called microbiologically influenced corrosion (MIC) caused by biofilms. This biocorrosion is usually associated with electrochemical effects caused by metabolic activity of the biofilm. Cristiani et al. (2002) gave a good overview of electrochemical techniques used in MIC monitoring, which is summarized below.

The open corrosion potential (OCP) is one of these techniques measured as the potential difference between a metal probe and a reference electrode. Several material combinations are used for that purpose, such as zinc and silver/silver chloride in seawater and copper/copper sulfate in soil, or stainless steel in cooling water systems (Mollica \& Cristiani 2003).

Electrochemical noise (EN) can also be measured at open circuits and detects fluctuations in potential or current. One critical aspect of this technique is the statistical evaluation and interpretation of the recorded signal noise.

Since no external current is applied in potential measurements like these, they do not change the biofilm as might be the case with other methods using electrical signals. 
Under a defined set of conditions both OCP and EN can indicate microbial activity and may even correlate with presence of biomass, but do not give direct and quantitative measurements (Nivens et al. 1995). Nevertheless, commercial MIC-monitoring equipment working with these methods is available (Pryfogle et al. 2002; Mollica \& Cristiani 2003).

Another group of electrochemical techniques are the analyte-selective microelectrodes with tip diameters in the range of micrometers, which have been used extensively to characterize chemical conditions within biofilms, such as $\mathrm{pH}$, and concentration of oxygen, sulfide, nitrate, and ammonium (Nivens et al. 1995).

Examples of analyte-selective microelectrode application are redox-potential electrodes for the monitoring of biofilm formation in recycling paper pulp (Holtmann \& Sell 2002), as well as pH and redox-potential electrodes for anoxic denitrifying biofilms (Li \& Bishop 2003). These investigations have been carried out under laboratory conditions. Since analyte-selective microelectrodes are fragile and their significant signal drift requires frequent calibration (Nivens et al. 1995), their use in field or industrial conditions appears less promising. As with OCP and EN measurements the results obtained with microelectrodes may indicate microbial activity and even correlate with presence of biomass under a defined set of conditions, but are inherently neither direct nor quantitative.

Electric resistance (ER) and linear polarization resistance (LPR) are techniques used in the monitoring of microbiologically influenced corrosion (MIC), and thus are focused on the chemical conditions associated with corrosion rather than on the biofilms which cause them. While the former merely measures accumulated corrosion rate, the latter yields a quantification of corrosion current, on which the biofilm's resistance has actually a counteracting effect (Cristiani et al. 2002). Both methods actively apply a current and thereby may change the biofilm, thus rendering results of continuous monitoring of a given sample questionable (Nivens et al. 1995).

\subsection{Impedance and capacitance measurements}

As far as electricity is concerned, living cells can be regarded as a closed, insulating membrane filled with liquid plasma that displays dielectric features.
This makes them behave like electric capacitors, capable of storing charges (i.e. ions that move towards the cell membranes) when exposed to an electric field. To be stored, however, these ions must move within the field, and since moving ions form an electric current, the electrical current flowing between two electrodes can be utilized to detect the presence of capacitance in the created field.

Since the ions move comparatively slowly in the plasma, the capacitance created by cells is relatively high at low frequencies but decreases at higher frequencies. This effect distinguishes between the cells' capacitance and the background capacitance of the surrounding medium. Typical frequencies used for that purpose are in the radiorange between 0.1 and $10 \mathrm{MHz}$.

The procedure of detecting biomass with this technique is designated Dielectric Spectroscopy, Electrical Admittance Spectroscopy or Capacitance Measurement, and was first described by Harris et al. as early as 1987. Commercial monitoring equipment of this kind is available, using a probe with four gold or platinum pins as electrodes. It is traditionally applied to monitor suspended biomass, like in industrial yeast fermentation processes, but there are also reports of its use in biofilm monitoring, though usually with weaker signals (Markx et al. 1990) and so far only in laboratory systems (Jass et al. 2001). The four-electrode arrangement does not allow high currents to flow, thus enabling measurements in media of high conductivity and excluding negative effects of electrode polarization, which have been observed in twoelectrode systems. Furthermore, the low electric currents have little or no effect on the biosystem under investigation.

Data presented by Markx et al. (1990) suggest that the detection limit of Dielectric Spectroscopy was in the order of $0.1 \mathrm{mg} \mathrm{cm}^{-2}$ in a pure Klebsiella rubiacearum-biofilm. Jass et al. (2001) reported biofilm detection in the range of $10^{8} \mathrm{cfu} \mathrm{cm}^{-2}$ in a mixed culture. An interesting feature of Dielectric Spectroscopy is that only living cells are capable of changing the system's capacitance, while the presence of lysed cells with disrupted membranes or inorganic matter usually yields no significant signals.

The kind of capacitance probes utilized in those works can be automatically cleaned by applying an electrical pulse that produces gas bubbles by electrolysis and can remove deposits from the elec- 
trodes, thus re-establishing the original condition (Markx et al. 1990).

Closely related to dielectric spectroscopy is a technique called electrochemical impedance spectroscopy (EIS). In this method the system's impedance is determined, which reduces the electric current with higher frequencies rather than increasing it like the capacitance. EIS, however, can only detect biofilms above $200 \mu \mathrm{m}$ of thickness, results are not easy to interpret and application is largely limited to laboratory conditions (Cristiani et al. 2002). Like in Dielectric Spectroscopy, however, the relatively small electrical perturbations leave activity and number of attached bacteria largely unaffected (Nivens et al. 1995).

\section{Vibration signals}

This section deals with methods that utilize mechanical vibrations in liquid or solid bodies to detect the presence of biofilms. Two different approaches must be distinguished here: sensors on which biofilms grow, with the vibrations being transmitted through the rigidity of the biofilm structure, and sensors that work like sonar, which create an acoustic pulse in the bulk liquid and monitor the echo from the walls and its modifications.

\subsection{Sensors on solid surfaces}

The sensors utilized for creating and measuring mechanical vibrations in direct contact with biofilms are usually piezoelectric crystals. These consist of a crystal or ceramic body with attached metal electrodes, and display a strong correlation between mechanical forces in the ceramic body and voltage between the electrodes. Since these interactions work in both ways, they can be used like microphones (as was already presented in the photoacoustic section) or for the detection of mechanical stress as in electronic balances, but also in the reverse sense as so-called actuators that directly transform electric signals into mechanical ones, like in loudspeakers or buzzers.

The principle of their use in chemical and biological analysis might be compared to the effect of knocking at several equal glasses with different amounts of water inside: the more water a glass contains, the lower will be the sound, since the additional mass reduces the resonance frequency of the mechanical system. Thus, by knocking and listening to the sound of a known glass, one can more or less precisely determine the amount of water inside.

The same effect occurs when biofilm attaches to the surface of a piezoelectric sensor and the extra mass affects the vibration properties. Rather than knocking on the sensor, the resonance frequency's modification is determined by electronically changing the frequency of the exciting electric voltage at the piezo electrodes and checking for the highest occurring current.

The best known piezo-sensor of this kind, which is also applied in biofilm monitoring, is the so-called quartz crystal microbalance (QCM, Nivens et al. 1993), also designated as thickness-shear mode quartz (TSM, Helle et al. 2000). In this device the whole surface vibrates transversally when alternating voltage is applied to the electrodes. Typical resonance frequencies are in the range of 5-10 MHz (Grate et al. 1993). Nivens et al. (1993) reported a detection limit for QCM of $3 \times 10^{5}$ cells $\mathrm{cm}^{-2}$ with a Pseudomonas cepaciabiofilm (Figure 6).

Since the rigidity of most biofilms is limited, the higher layers of a thick biofilm would not contribute to that result. They can rather be detected by measuring the dissipation or frictional energy loss induced, which corresponds to the vibration's continuation after switching off the exciting voltage (Rudh et al. 2002), or an increase in impedance of the electric system (Hartmann et al. 2002).

It should be mentioned that QCMs are affected by the environmental temperature as well as the liquid phase pressure as was reported by Nivens et al. (1993). Compensation procedures for both should be provided in the equipment if necessary.

Several other kinds of piezoelectric devices are applied in other fields. They differ in the

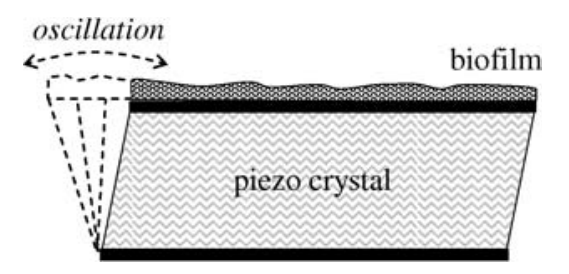

Figure 6. Cross-section of an oscillating QCM. 
arrangement of their electrodes and the piezoelectric orientation of the crystal body, thus creating and detecting different kinds of vibrations. Examples include: surface acoustic wave (SAW) devices, in which just the surface is corrugated vertically, flexural plate wave (FPW) devices, in which the whole plate corrugates vertically, and acoustic plate mode (APM) devices, in which the surface is distorted horizontally (Grate et al. 1993). Though such devices are used extensively in chemical analysis, no information about their application in biofilm monitoring is yet available.

\subsection{Sensors in the liquid phase}

Smooth surfaces create strong acoustic echoes, while soft and patchy structures like biofilm surfaces attenuate sound effectively. This fact is utilized by a biofilm monitoring technique designated ultrasonic frequency-domain reflectometry (UFDR), which was described by Fonseca et al. (2002). In this work ultrasound signals in the range between 14 and $24 \mathrm{MHz}$ were emitted in the bulk liquid in front of a polycarbonate substrate. Their echo was recorded and analyzed to detect the formation of a Pseudomonas aeruginosa biofilm on the substrate.

A correlation between reflection amplitude and exopolysaccharides (EPS) concentration, which was used to estimate biofilm coverage on the surfaces, could be established, once the biofilm had reached a concentration of $35 \mu \mathrm{g} \mathrm{cm}^{-2}$.

\section{Conclusions}

It may be regarded as a main conclusion to be drawn from the preceding overview, that for each goal in online biofilm monitoring there exist other suitable monitoring methods. A universal method capable of yielding all interesting information has not yet been and probably will never be found.

Thus, the first step in choosing the best suited online biofilm monitoring method should be to determine exactly which kind of detection limit is needed and which features or parameters are of interest:

- If the main consideration is the obstruction of flow or heat transfer or plugging of installations by biofilms, their internal features might be neglected and some representative tubing or small models of heat exchangers can be used to directly derive the necessary information (see Sections 2.3 and 2.4). Alternatively the presence and thickness of deposits including biofilms can be monitored by optical methods (2.1 and 2.2), quartz crystal microbalances (7.1) or electrical impedance and capacitance (6.2), given that the deposit can reach a certain thickness before being detected.

- If certain metabolic activities are important, one might concentrate on their determination either directly by means of the respective metabolic products (Section 3), or - depending on the situation in question - indirectly by bioluminescence (5.1), fluorometry (5.2), spectroscopy (5.3), attenuated total internal reflection (ATR-EW, 5.4), or electrochemical techniques such as MIC-electrodes (6.1).

- If both the physical structure and chemical properties are of interest, nuclear magnetic resonance (NMR, 5.5) and photoacoustic spectroscopy (PAS, 5.6) might be taken into consideration.

- For the monitoring of relatively thin biofilms (i.e. in the range of $10^{5}$ cells $\mathrm{cm}^{-2}$ ) quartz crystal microbalances (7.1) may be considered as well as fiber optical devices (2.2) and attenuated total internal reflection (ATR-SPR and ATR-EW, 5.4), depending on the biofilms' optical properties.

- For a very high resolution down to individual cells one might actually take microscopic techniques and computerized image analysis (4) into consideration if local conditions allow for the use of such equipment.

Practically all the described techniques are noninvasive and allow continuous monitoring of biofilm formation. Automatic data collection is easy to implement. This permits the set up of realtime, on-line methods for preventing biofilm formation or for cleaning fouled surfaces (which are frequent in industry) and opens challenging opportunities for the development of expert system control techniques based on artificial intelligence concepts.

Table 1 summarizes the individual methods' characteristics. Given the number of factors and parameters concerned, however, this obviously must be limited to a qualitative overview. 


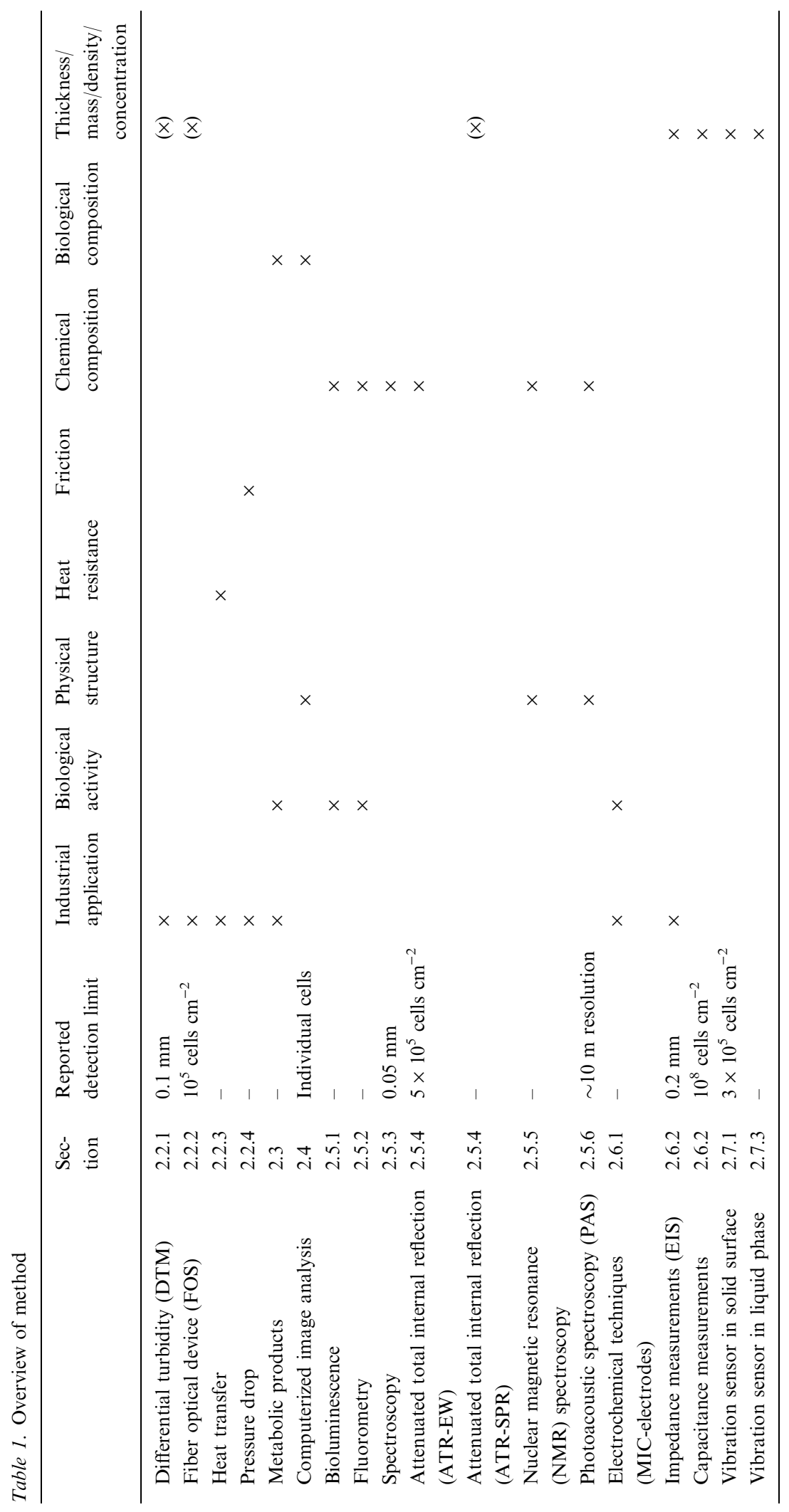




\section{References}

Angell P, Arrage AA, Mittelmann MW \& White DC (1993) Online, non-destructive biomass determination of bacterial biofilms by fluorimetry. J. Microbiol. Meth. 18: 317-327

As HV \& Lens P (2001) Use of 1H NMR to study transport processes in porous biosystems. J. Ind. Microbiol. Biotechnol. 26: 43-52

Bastos F, Mesquita RBR, Ferreira Jorge RM, Fernandes SMV, Castro PML \& Rangel AOSS (2002) On-line monitoring of a trickling filter during treatment of chlorobenzene contaminated waste. Proceedings of the International Specialized Conference on Biofilm Monitoring, Porto, March 17-20, 218-221

Carrión M, Asaff A \& Thalasso F (2003) Respiration rate measurement in a submerged fixed bed reactor. Water. Sci. Tech. 47(5): 201-204

Cristiani P, Perboni G, Hilbert L, Mollica A \& Gubner R (2002) Experiences on MIC monitoring by electrochemical techniques. Proceedings of the International Specialized Conference on Biofilm Monitoring, Porto, March 17-20, $197-200$

Characklis WG, Turakhia MH \& Zelver N (1990) Transport and interfacial transfer phenomena. In: Characklis WG, Marshall K (Eds) Biofilms (pp 265-340). John Wiley \& Sons, New York

Chenoweth JM (1988) Liquid fouling monitoring equipment. In: Melo LF, Bott TR \& Bernardo CA (Eds) Fouling Science and Technology (pp 49-65). Kluwer Academic Publisher, Dordrecht

Festy D, Tribollet B \& Monfort-Moros N (2002) A sensor for $\mathrm{C}$-steel MIC assessment in SRB environment. Proceedings of the International Specialized Conference on Biofilm Monitoring, Porto, March 17-20

Fonseca AC, Greenberg AR \& Hernandez M (2002) Real time biofilm detection using ultrasonic frequency-domain reflectometry (UFDR). Proceedings of the International Specialized Conference on Biofilm Monitoring, Porto, March 17-20

Freitas dos Santos LM \& Livingston AG (1995) Membraneattached biofilms for VOC wastewater treatment. I: Novel in situ biofilm thickness measurement technique. Biotechnol. Bioeng. 47(1): 82-89

Göransson A \& Trägårdh C (2000) An experimental study of the kinetics of particle deposition in a wall-jet cell using total internal reflection microscopy. J. Coll. Interf. 231: 228-237

Grate JW, Martin SJ. \& White RM (1993) acoustic wave microsensors part I Anal. Chem. 65(21): 940-995

Harris CM, Todd RW, Bungard SJ, Lovitt RW, Morris JG \& Kell DB (1987) The dielectric permittivity of microbial suspensions at radio frequencies: A novel method for the real-time estimation of microbial biomass. Enzyme Microbial Technol. 9: 181-186

Hartmann J, Teichmann L, Horn H, Borngräber R, Lucklum R \& Hauptmann P (2002) Quartz crystal microbalance for online-early-diagnosis of growing biofilms. Proceedings of the International Specialized Conference on Biofilm Monitoring, Porto, March 17-20

Helle H, Vuoriranta P, Välimäki H, Lekkala J \& Aaltonen V (2000) Monitoring of biofilm growth with thickness-shear mode quartz resonators in different flow and nutrition conditions. Sensors and Actuators. part b, chemical 71(1/2): $47-54$

Heydorn A, Nielsen AT, Hentzer M, Sternberg C, Givskov M, Ersboll BK \& Molin S (2000) Quantification of biofilm structures by the novel computer program COMSTAT. Microbiology 146: 2395-2407

Holtmann D \& Sell D (2002) Investigations into the application of a process for the determination of microbial activity in biofilms. Proceedings of the International Specialized Conference on Biofilm Monitoring, Porto, March 17-20

Jass J, ONeill JG \& Walker JT (2001) Direct biofilm monitoring by a capacitance measurement probe in continuous culture chemostats, Methods Enzymol. 337: 63-69

Kappelhof JWNM, Vrouwenvelder HS, Schaap M, Kruithof JC, van der Kooij D \& Schippers JC (2002) An in situ biofouling monitor for membrane systems. Proceedings of the 5th Conference on Membranes in Drinking and Industrial Water Production (MDIW), Mülheim/Ruhr, September 2226, 2002

Klahre J \& Flemming HC (2000) Monitoring of biofouling in papermill process waters Wat. Res. 34(14): 3657-3665

Knudsen JG (1981) Apparatus and techniques for measurement of fouling of heat transfer surfaces. In: Somerscales EFC \& Knudsen JG (Eds) Fouling of Heat Transfer Equipment ( $p$ 57). Hemisphere Publishing Corporation, Washington

Le Bras S, Festy D, Lacotte N \& Le Haitre M (1998) A non destructive method for biofilm monitoring on optical systems. Proceedings, Ocean's 98 IEEE Conference and Exhibition, Nice, September 28-October 1

Leitz M, Tamachkiarow A, Frankel H \& Grattan KTV (2002) Monitoring of biofilm growth using ATR-leaky mode spectroscopy, J. Phys. D: Appl. Phys. 35: 55-60

Lewandowski Z \& Beyenal H (2003) Biofilm monitoring: A perfect solution in search for a problem. Water Sci. Technol. 47(5): 9-18

Li J \& Bishop PL (2003) Monitoring the influence of toxic compounds on microbial denitrifying biofilm processes. Water Sci. Technol. 47(5): 211-216

Markx GH \& Kell DB (1990) Dielectric spectroscopy as a tool for the measurement of the formation of biofilms and their removal by electrolytic cleaning pulses and biocides. Biofouling 2: 211-227

Mollica A \& Cristiani P (2003) On-line biofilm monitoring by "BIOX" electrochemical probe. Water Sci. Technol. 47(5): 45-49

Nivens DE, Palmer RJ \& White DC (1995) Continuous nondestructive monitoring of microbial biofilms: a review of analytical techniques. J. Ind. Microbiol. 15: 263-276

Nivens DE, Chambers JQ, Anderson TR \& White DC (1993) Long-term, on-line monitoring of microbial biofilms using a quartz crystal microbalance. Anal. Chem. 65: 65-69

Pereira MA, Roest K, Stams AJM, Akkermans ADL, Amaral AL, Pons MN, Ferreira EC, Mota M \& Alves MM (2003) Image analysis, methanogenic activity measurements and molecular biological techniques to monitor granular sludge from an EGSB reactor fed with oleic acid. Water Sci. Technol. 47(5): 181-188

Philip-Chandy R, Scully PJ, Eldridge P, Kadim HJ, Grapin G, Jonca MG, D'Ambrosio MG \& Colin F (2000) An optical fiber sensor for biofilm measurement using intensity modulation and image analysis. IEEE J Sel Top Quantum Electron 6(5): 764-772

Pinheiro MM, Melo LF, Bott TR, Pinheiro JD \& Leitao L (1988) Surface phenomena and hydrodynamic effects on the deposition of Pseudomonas fluorescens. Can. J. Chem. Eng. 66: 63-67

Pryfogle PA, Mines GL, Sperry TL \& Allred RG (2002) Investigation of an electrochemical monitor for tracking 
biofilm development at the Bonnett Geothermal Plant, Cove Fort, Utah. Presented at the Geothermal Resources Council (GRC) meeting in Reno, Sept. 22-25, 2002

Rudh M, Green H, Lie E \& Sjöström L (2002) Measuring biofilm formation in real-time by quartz crystal microbalance with dissipation monitoring (QCM-D) Proceedings of the International Specialized Conference on Biofilm Monitoring, Porto, March 17-20

Saxena I, Sturman PJ \& Costerton JW (2002a) Development and testing of a fiber-optic probe for biofilm detection and quantification. Proceedings of the International Specialized Conference on Biofilm Monitoring, Porto, March 17-20

Saxena I, Files D, Rao S \& Costerton WJ (2002b) Autofluorescence-based bacteria detection using an optical fiber, Proceedings of SPIE - The International Society for Optical Engineering v 4622, 106-111

Schmid T, Kazarian L, Panne UM \& Niessner R (2001) Depthresolved analysis of biofilms by photoacoustic spectroscopy. Anal. Sci. 17(Special Issue): 574-577

Schmid T, Panne U, Haisch C, Hausner M \& Niessner R (2002) A new photoacoustic technique for depth-resolved in situ monitoring of biofilms. Environ. Sci. Technol. 36(19): 41354141

Schmid T, Panne U, Haisch C \& Niessner R (2003) Photoacoustic absorption spectra of biofilms. Rev. Sci. Instrum. 74: $755-757$
Tamachkiarow A \& Flemming H-C (2003) On-line monitoring of biofilm formation in a brewery water pipeline system with a fibre optical device. Water Sci. Technol. 47(5): 1924

Tinham P \& Bott TR (2003) Biofouling assessment using an infrared monitor. Water Sci. Technol. 47(5): 39-43

Vanhooren H, Van Hulle S, De Pauw D \& Vanrolleghem PA (2002) Monitoring and modelling a pilot-scale trickling filter using on-line off-gas analysis. Proceedings of the International Specialized Conference on Biofilm Monitoring, Porto, March 17-20

Wetegrove RL \& Banks RH (2000) Optical fouling monitors. Biocorrosion Network - Workshop on Monitoring Systems, Venezia, April 12-14

Wolf G, Crespo JG \& Reis MAM (2002) Optical and spectroscopic methods for biofilm examination and monitoring, Review Environ. Sci. Biotechnol. 1: 227-251

Wolf G, Almeida JS, Crespo JG \& Reis MAM (2003) Monitoring of biofilm reactors using natural fluorescence fingerprints, Water Sci. Technol. 47(5): 161-167

Xavier JB, Malhó R, Reis MAM \& Almeida JS (2000) Description of biofilm formation by determination of developmental axis. Water Sci. Technol. 41(4-5): 121-127

Xavier JB, White DC \& Almeida JS (2003) Automated biofilm morphology quantification from confocal laser scanning microscopy imaging. Water Sci. Technol. 47: 31-37 